\title{
Chimneys in the starburst galaxy M 82
}

\author{
Matt P. Redman, K.A. Wills, T.W.B. Muxlow and A. Pedlar \\ Department of Physics \& Astronomy, University of Manchester, \\ Manchester M20 1QE, UK
}

\begin{abstract}
A new radio image of the core of the starburst galaxy M 82 is presented. A pair of probable galactic chimneys are evident. Such structures have been conjectured to result when a giant shell of ionized gas breaks out of the dense plane of a galaxy and ejects hot gas into the halo. In M 82, the starburst activity drives a galactic superwind and theoretical models of this process require a knowledge of the kinematics of the hot gas in the core of the galaxy. The results presented here imply that the ejection of material into the base of the superwind does not occur smoothly over the starburst region; instead, very localised venting of the hot gas appears to be taking place.
\end{abstract}

When massive stars are found in rich enough OB associations, the giant shells that result from the combined action of their winds and UV radiation eventually become predominantly driven by supernova explosions and Wolf-Rayet stellar winds. Giant shells can attain sizes of several tens or even hundreds of parsecs. If their size becomes comparable to the characteristic ISM density scale height of the disk gas then the shells become elongated in the direction perpendicular to the galactic plane. The hot low density gas internal to the giant shell can eventually 'blow out' through a chimney into the galactic halo (Norman \& Ikeuchi 1989).

Our new $5 \mathrm{GHz} V L A$ observations show evidence of a pair of such chimneys emerging from the region containing the brightest SNR $41.95+57.5$ and has previously been shown to be the site of a giant $\mathrm{H}$ II region (Wills et al. 1997; Achtermann \& Lacy 1995). This result bears a striking similarity to the chimney observed in our own galaxy by Normandeau et al. (1996). To the south we see a 'cone' of missing continuum emission with a large opening angle and to the north we observe a smaller cone containing weak continuum emission. Together with previous observations, our new imagery appears to show an excellent example of a chimney flow produced by the expansion of a giant $\mathrm{H}$ II region into the density gradient perpendicular to the plane of the galaxy.

\section{References}

Achtermann, J.M., Lacy, J.H. 1995, ApJ 439, 163

Norman, C.A., Ikeuchi, S. 1989, ApJ 345, 372

Normandeau, M., Taylor, A.R., Dewdney, P.E. 1996, Nature 380, 687

Wills, K.A., Pedlar, A., Muxlow, T.W.B., Wilkinson, P.N. 1997, MNRAS 291, 517 


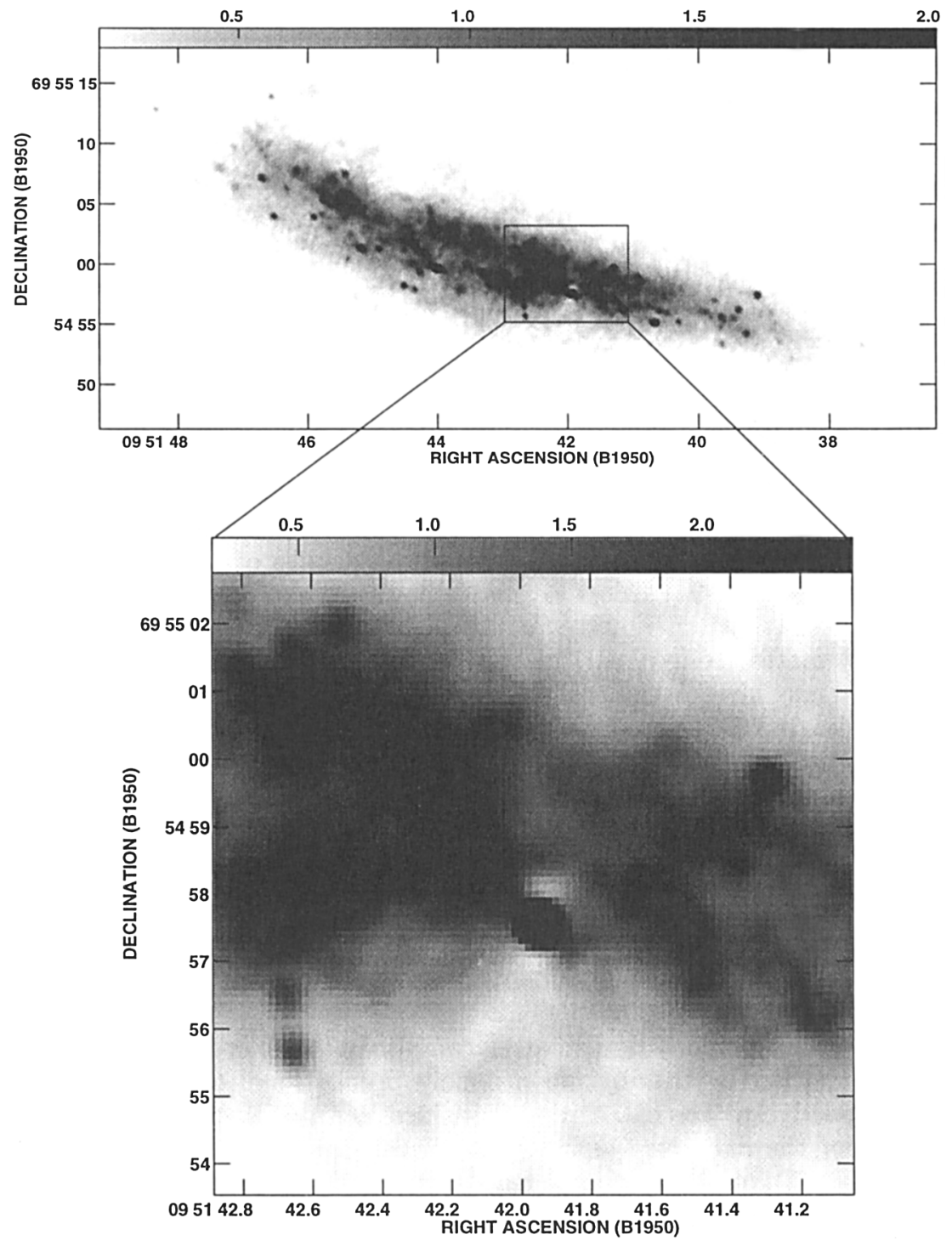

Figure 1. VLA A+B array $5 \mathrm{GHz}$ image of $\mathrm{M} 82$ with 350 mas resolution. The boxed enlargement shows the chimney features. The bright compact sources in this image are supernova remnants. 